\title{
Mini-Review Article - Current Opinion on Salivary Biomarkers as a Measurement for Stress and Fatigue
}

\author{
Ting Chun $\mathrm{Yi}^{\mathrm{a}}$, and Shabbir Moochhala ${ }^{\mathrm{b}, \mathrm{c}, *}$ \\ ${ }^{a}$ Faculty of Medicine, Nursing and Health Sciences, Monash University, Victoria 3800, Australia \\ ${ }^{b}$ Department of Pharmacology, Yong Loo Lin School of Medicine, Clinical Research Centre \#05-09, 10 Medical Drive, \\ 117597, Singapore \\ ${ }^{c}$ DSO National Laboratories, 27 Medical Drive \#09-01, 117510, Singapore
}

\begin{abstract}
Salivary biomarkers have been increasingly popular in stress research as saliva is easily produced and collection is non-invasive and not limited by geographical distance or lack of infrastructure. Several salivary biomarkers have been utilized in stress research, for instance, salivary cortisol, salivary amylase and salivary immunoglobulin A. Despite being sensitive to changes in fatigue, they have limitations such as inter-individual variability, and interactions with other constituents that may confound the results. Recently, Hyperion Biotechnology has developed the Fatigue Biomarker Index (FBI), which is a measurement of the changes in concentration of salivary peptides with fatigue. The FBI has been shown to be an accurate and objective biomarker of fatigue, and has huge potential for use in various fields and industries.
\end{abstract}

This article will review some of the previous and current salivary biomarkers of stress, as well as critically appraise the new salivary peptide test in terms of its accuracy, application and access.

Keywords: Fatigue, Hyperion, saliva, salivary biomarker, stress.

\section{INTRODUCTION}

Fatigue is something familiar to everybody; it affects everyone, regardless of gender, race, occupation and social status. It creeps into our lives, and has been identified as the leading preventable cause of transportation accidents [1]. It is also a significant manifestation of many disease processes including psychiatric disorders like depression, malignancy and autoimmune diseases [2].

For many years, researchers have been searching for a biomarker that can objectively measure the amount of stress or fatigue one is facing. This would help facilitate the prevention of fatigue in certain jobs and excessive physical activity, and verify the effects of medications and supplements. In the past several years, salivary biomarkers have been assessed, however, they have limitations in terms of accuracy.

Hyperion Biotechnology has recently discovered a method to measure the relationship between the composition of certain saliva peptides and fatigue, and hence objectively measure one's fatigue level using the Fatigue Biomarker Index (FBI).

*Address correspondence to this author at the Department of Pharmacology, Yong Loo Lin School of Medicine, Clinical Research Centre \#05-09, 10 Medical Drive, 117597, Singapore; Tel: +654857201; Fax: +654857226;

E-mail : mshabbir@dso.org.sg

\section{PREVIOUS AND CURRENT MARKERS OF FA- TIGUE}

\section{Traditional Markers of Fatigue}

Traditionally, fatigue is measured based either on mood state eg. Profile of Mood States (POMS), neurobehavioural assay e.g. Psychomotor Vigilance Task, or a physiological marker eg. Interleukin-6 (IL-6) levels in blood [1]. However, all these measurements have their limitations.

For instance, as POMS involves a questionnaire, it may be subjective and some individuals may report their fatigue levels falsely, especially in certain industries where there are incentives to appear less tired [1]. The Psychomotor Vigilance Task has a standard duration of 10 minutes, and may not always be practical, especially in time-constrained circumstances [3].

Physiological markers that measure the level of particular substances in blood have been widely used. For instance, IL6 is shown to be at higher levels in fatigued breast cancer survivors as compared to non-fatigued survivors [4]. Besides, CD26, a receptor on immune cells, is a good indicator of the function of natural-killer cells, and has been used to aid diagnosis of chronic fatigue syndrome or fibromyalgia [5]. However, these tests are invasive and cause discomfort to the subjects, and require trained healthcare professionals to collect blood specimens. 


\section{SALIVARY BIOMARKERS OF FATIGUE}

Salivary biomarkers have gained popularity in stress research as it has proven superior to testing biomarkers in blood. With recent advances in the field of immunological and molecular biology, scientists have found more promising ways of analyzing salivary biomarkers and apply them to stress research [6].

There are many advantages with using salivary biomarkers as compared to blood in stress research. Firstly, it is non-invasive, eliminates the risk of needle-stick injuries and causes minimal distress to individuals [1]. The stress from the fear of needles during collection, which may bias the results, is also minimized [7]. This makes it useful in certain populations, such as children, in which phlebotomy may be traumatic. Salivary biomarkers are also useful for research involving healthy children as it avoids ethical concerns associated with invasive measures [8].

Besides, samples can be obtained at short intervals as saliva is constantly being produced [8].

Furthermore, saliva collection has does not require laboratory facilities or skilled healthcare professionals. This makes it highly useful in field research or ambulatory assessments in the subjects' natural environment [8].

Moreover, samples can be collected just prior to a stressful event, without the need of being in a hospital or requiring any equipment apart from a collection device. Saliva can even be collected in remote geographical areas where access to healthcare is limited or where phlebotomists are unavailable [6].

Currently, several salivary biomarkers have been used in stress research, for instance, salivary cortisol, salivary amylase and salivary immunoglobulin A levels (see Table 1).

\section{Salivary Cortisol}

Salivary cortisol has been used as a biomarker of both mental and physical stress, as cortisol has been regarded as a reliable measure of the hypothalamic-pituitary-adrenal (HPA) axis adaptation to stress.

In times of stress, the HPA axis responses by stimulating the production of adrenocorticotrophic hormone (ACTH) that leads to increased secretion of cortisol from the adrenal glands into the blood [8]. In the blood, most cortisol is bound to carriers, with only $1-15 \%$ of cortisol being free and biologically active [9]. The free fraction of cortisol can be measured in saliva, and this reliably reflects the amount of free cortisol circulating in the blood [2,8]. Salivary cortisol has been shown to be sensitive in detecting HPA axis dysregulation related to stress and disease, including chronic fatigue syndrome [2].

\section{Advantages}

Firstly, the level of free cortisol in saliva corresponds well to that in blood, and is independent of transport mechanisms, salivary enzymes and salivary flow rate. The correlation between salivary and free serum cortisol levels is more than $80 \%[8]$.
Besides, cortisol is transferred from plasma to saliva within a few minutes [8], and this short time lag is useful in stress research.

Cortisol is also relatively stable in saliva, thus saliva samples can be stored at room temperatures for at least for weeks [8], meaning that samples can be taken anywhere and then mailed to a laboratory with the relevant facilities, thus enabling ambulatory studies to be conducted.

\section{Limitations}

As mentioned earlier, salivary cortisol only measures the free fraction of cortisol, and thus does not completely reflect the total plasma cortisol levels. The free (unbound) cortisol is dependent on levels of cortisol-binding globulins (CBG), which may be affected by certain conditions, for example, pregnancy, menstrual cycle and consumption of oral contraceptive pills (OCP] [10]. For instance, OCP increases CBG levels, which increase the number of cortisol binding sites in the circulating blood, resulting in decreased free cortisol [8].

Once CBG is saturated, the linear relationship between free and total cortisol levels in blood is altered. Therefore, factors that affect CBG levels ultimately influence the linear relationship between salivary and total cortisol (10), thus reducing the reliability of salivary cortisol as a reflection of total serum cortisol levels.

In addition, within the general population, there exists an interpersonal variability with regards to the sensitivity of glucocorticoids, meaning that different people may respond differently to a same level of cortisol [8]. Some of these confounding factors include gender, substance use, steroid supplementation or hormone replacement therapy, and pregnancy [11]. For instance, males have a larger salivary cortisol response following short-term laboratory stress as compared to women [11]. Chronic smoking or nicotine exposure as well as chronic alcohol consumption leads to reduced salivary cortisol response to stress due to blunting of the HPA axis response to stress [11].

Nevertheless, salivary cortisol is still a useful biomarker in stress research, but it is important that the possible sources of variance and confounding factors are being considered [10].

\section{Salivary $\alpha$ Amylase (sAA)}

Salivary $\alpha$-amylase (sAA) is a digestive enzyme involved in the breakdown of starch. sAA is synthesized in acinar cells and stored in granules and released into saliva in response to neuronal stimuli [12]. During stressful situations, the sympathetic nervous system is stimulated, and results in increased sAA secretion by the salivary glands. Therefore, sAA has been used as a biomarker for stress [2].

\section{Advantages}

sAA has demonstrated high sensitivity to stress-related changes, including both psychological stress (for example, undergoing medical procedures) and physical stress (for example, exercise) [12]. During exercise, there is an increase in sympathetic activity, causing increased adrenergic activity in the salivary glands, resulting in a marked increase in sAA levels in saliva following exercise [12]. Chatterton et al con- 
ducted trials on subjects undergoing running and bicycle exercises, and observed significant increases of sAA after exercise $[12,13]$. They also demonstrated a dose-response relationship, with the highest increases in sAA corresponding to running as compared to light jogging and walking [13].

\section{Limitations}

One important confounding factor in using sAA as a measurement of fatigue is that the parasympathetic nervous system (PSNS) also plays a role in sAA secretion by increasing salivary flow rate [14]. However, sympathetic nervous system [SNS] stimulation results in PSNS inhibition, causing a reduction in salivary flow rate and thereby decreasing saliva production. As such, the increased sAA concentration measured in stress might be partly attributed to a decreased salivary volume [2].

Besides, there are numerous salivary glands that contribute to the salivary protein content and thus produce sAA as well. These salivary glands have varied response to autonomic and mechanical stimulation. Mechanical stimulation (ie. Chewing) shifts the balance from submandibular gland secretion (modest sAA concentration) to parotid gland secretion (high sAA content) that is independent of central SNS regulation [14], therefore casting doubt on the reliability of sAA as a biomarker of fatigue and stress.

Moreover, sAA levels are influenced by numerous factors which may lead to inter-individual variability, thus once again undermining the accuracy of sAA as a biomarker for fatigue. For instance, studies have shown that cigarette smoking decreases basal $\alpha$-amylase activity in saliva and that people who chronically drink alcohol have lower amylase levels [13]. Also, certain somatic diseases are associated with either higher or lower sAA concentrations. For instance, asthma and some autoimmune conditions appear to lower sAA levels, whereas chronic obstructive pulmonary disease (COPD) seems to raise sAA levels.

\section{Salivary Immunoglobulin A}

Salivary secretory immunoglobulin A (sIgA) can be used as an indirect biomarker of fatigue. Salivary sIgA is found on mucous membranes and is involved in the immune response against pathogens [15]. Decreased sIgA concentrations correlates with immune suppression [15].

Evidence suggests that intense physical exercise leads to a transient suppression of $\operatorname{IgA}$ and long-term regular intense physical activity, especially in adverse conditions, results in chronic suppression of sIgA [15]. As such, sIgA can be used as a salivary marker of fatigue secondary to immune suppression from physical stress.

\section{Advantages}

The efficacy of $\operatorname{sIgA}$ as a biomarker has been tested on wildlife firefighters, and results demonstrated that $\operatorname{sIgA}$ was suppressed in response to extended workshifts, and that $\operatorname{sIgA}$ levels are sensitive to fatigue secondary to extreme working conditions [16].

\section{Limitations}

There is huge biological variation and inter-individual variability in sIgA levels, as well as diurnal variation, all of which reduce the validity of $\operatorname{sg} \mathrm{A}$ as a biomarker of stress [15].

Besides, sIgA appears to be less sensitive when compared with other biomarkers of stress. A study was conducted to determine the effects of a Mind-Body Medicine Skills [MBMS] course to medical students in a university in United States using several biomarkers including cortisol, sIgA and testosterone. While cortisol and testosterone levels displayed significant changes, sIgA levels were not statistically different [17].

\section{THE NEW SALIVARY BIOMARKER - SALIVARY PEPTIDE TEST}

The above salivary biomarkers described, although show correlation with fatigue, have their limitations and are unable to provide an objective marker of one's level of fatigue. Hyperion Biotechnology has recently developed a new technology that uses the changes in concentration of salivary peptides to create an objective biomarker for fatigue.

Hyperion Biotechnology found that fatigue altered the composition of the small molecular weight proteome of saliva [1]. This is based on the physiological principle that since salivary glands are innervated by neurons from both SNS and PSNS, changes in fatigue lead to quantifiable changes in saliva composition [1]. Using reversed-phase liquid chromatography and high resolution mass spectrometry, the various components of processed saliva are separated and elucidated. The ratio of concentrations for 2 peptide fragments in saliva that change in opposite directions with fatigue is calculated. This ratio is referred to as the Fatigue Biomarker Index [FBI] and can be used to track changes in fatigue level [1].

A double-blind, random crossover trial was conducted on 9 male recreationally trained male cyclists ranging from 1946 years old. The subjects underwent an exercise program and saliva samples were collected at regular intervals during the exercise sessions. After each exercise type, the participants also evaluated their relative perceived exertion (RPE) [1]. Results showed that FBI decreased with time as RPE increased [1], and hence demonstrates that these salivary peptides can be used as an objective biomarker for evaluation of stress and fatigue.

\section{DISCUSSION AND CRITICAL APPRAISAL}

The Fatigue Biomarker Index is a simple yet sophisticated method of quantifying fatigue in individuals. Due to its objectivity, the Fatigue Biomarker Index has many advantages over other salivary biomarkers of stress and fatigue, and is considered a breakthrough in fatigue measurement.

\section{ACCURACY}

Two important aspects of a successful biomarker are reliability and validity. In the abovementioned study, the trial 
was conducted on a very small subject population of 9 male cyclists, who were aged between 19-46 years. As such, it is necessary to ascertain as to whether the results would be reproducible in a larger population with more interpersonal variability.

The FBI has been tested in more than 200 healthy, young adults in the military and results showed that the FBI varied by more than 10,000-fold between fatigued and well-rested individuals [18], and thus this adds proof that the FBI can be reliably used as an objective biomarker of fatigue.

As the FBI is still a relatively new player in the market, there are still many aspects about its properties and possible confounding factors that need to be investigated. Current data has yet to provide clear evidence as to whether the FBI, like $\operatorname{sgA}$, shows diurnal variation and whether it, like salivary cortisol and sAA, shows significant inter-individual variability. Therefore, more comprehensive studies involving larger populations of subjects from various demographics (ie. Larger age range, people with a wider range of fitness levels) would have to be conducted in order to validate that FBI is a reliable and objective biomarker of fatigue.

\section{APPLICATION}

The invention of the FBI enables identification of a fatigued state, monitoring the level of fatigue over time with physical activity and even the identification of a decrease in physical performance capability over time [19]. Besides, it also enables identification of recovery from a fatigued state in a subject [19].

As such, the FBI would be especially useful in the sporting and military fields in which it can be used to explore and quantify the effects of sleep deprivation or the effects of prolonged exercise on soldiers or athletes. Analysis of this valuable data might then lead to development of more optimized training regimens which are better suited for each individual. It can also be used to monitor the effects of supplements and lifestyle modifications (for instance, changes in diet, sleep) on one's fatigue level. In fact, the FBI can be used by anyone to obtain a more quantitative idea on their current level of stress and fatigue, and hence modify aspects of their life in order to optimize their health [20].
Besides, the FBI has been utilized as a tool for the prediction of success in intensive training programs for instance, special forces in the military. If successful, it may be utilized as a guide in selection of people suitable for these training programs, and the candidates chosen would be suitable for the program and have a higher chance of successfully completing it [20].

In the healthcare and pharmaceutical industry, there is potential to use the FBI to more extensively investigate the effects of medications and supplements in terms of their efficacy and effectiveness, and possibly lead to further pharmaceutical development. This is especially so for antidepressants, which show a positive placebo effect in about $40 \%$ of people who are depressed [20]. The FBI may perhaps even lead to the development of effective anti-fatigue medications, which would greatly benefit many people, especially those suffering from chronic diseases and chronic fatigue syndrome. Also, the FBI will aid research in quantifying the impact of certain somatic and psychiatric diseases on lifestyle and increase our limited understanding of these diseases.

Moreover, the FBI would also be a valuable tool in assessing the impact of certain jobs on health, especially shift workers, junior doctors, caregivers for the elderly or the sick. Junior doctors tend to suffer from sleep deprivation due to long working hours, rotating extended shifts and overnight call duty. It is found that sleep deprivation not only affects one's health, it also results in decreased clinical performance and increased risk of medical mistakes, which may cause harm to patients [21]. In this circumstance, the FBI would act as a good marker to quantify the impact of sleep deprivation on performance and perhaps even establish the ideal number of working hours in order to maximize performance.

A retired American Air Force colonel had trialed the saliva test by ascertaining the effect of fatigue on air traffic controllers being trained for a mission, and found it a simple, accurate and convenient test [22]. With such successes, this may potentially lead to changes in infrastructure, support or policies with an aim to optimize working conditions that will result in raised workplace productivity. These changes may largely impact these workers and their clients, and may have

Table 1. Summary of Available Salivary Biomarkers

\begin{tabular}{|c|c|c|}
\hline Salivary Biomarker & Advantages & Limitations \\
\hline Salivary Cortisol & $\begin{array}{ll}\text { - } & \text { Level corresponds well to that of blood } \\
\text { - } & \text { Short lag time } \\
\text { - } & \text { Relatively stable and can be stored for sev- } \\
\text { eral weeks }\end{array}$ & $\begin{array}{ll}\text { - } & \text { Only measures free fraction of cortisol } \\
\text { - } & \text { Influenced by conditions that affect CBG levels } \\
\text { - } & \text { Inter-individual variability }\end{array}$ \\
\hline Salivary $\alpha$-Amylase & $\begin{array}{l}\text { High sensitivity to stress related changes, } \\
\text { with dose-response relationship }\end{array}$ & $\begin{array}{ll}\text { - } & \text { Salivary flow rate also affected by PSNS } \\
\text { - } & \text { sAA production is dependent on numerous salivary } \\
\text { glands that have different responses to stimulation } \\
\text { - } \quad \text { Inter-individual variability }\end{array}$ \\
\hline Salivary Immunoglobulin A & - $\quad$ Sensitive marker of fatigue & $\begin{array}{l}\text { - } \quad \text { Huge biological variation and inter-individual variability } \\
\text { - } \quad \text { Not as sensitive as other biomarkers of stress }\end{array}$ \\
\hline
\end{tabular}


a multiplier effect on society. The FBI may even impact the economy due to less days lost from absences from work.

Furthermore, invention of the FBI also provides correlation of a subject's perceived level of fatigue with that of an objective biomarker, and hence can be used in the development of other qualitative measurements of fatigue [19], possibly resulting in further advancements in the research of fatigue.

\section{ACCESS}

In terms of stress research, using saliva as a biomarker for fatigue is highly convenient, non-invasive and readily available, making it an excellent biomarker. The collection of saliva is also very simple and does not require any skills or expertise, making it widely accessible, as it can even be done in the comfort of one's home.

Based on the invention of the FBI, a home testing kit for the measurement of fatigue has been developed. This kit is highly accessible by the general public and simple to use. It only requires collection of $15 \mathrm{ml}$ of saliva in a tube, which is then mailed to the Hyperion Biotechnology lab. Results will be available within one week [20].

One limiting factor of the FBI would be cost. The test kits are priced from $\$ 55$ to $\$ 125$ depending on the number of tests ordered [22]. Even though the price is not exorbitant, it may be considered expensive by some people. However, it can be argued that the value of knowing one's fatigue level has very useful implications, depending on the reason for the test, and this may outweigh the cost required for the test.

Besides, in order to process saliva and measure FBI, a laboratory with relatively advanced level of technology that supports equipment like liquid chromatography and mass spectrometry would be required. The construction of such a laboratory would also require large amounts of funding, and may not be available in certain less developed areas. Currently, work is being done to identify and develop antibodies against the salivary peptides. If successful, this would simplify the detection and elucidation process and potentially lower the cost.

\section{CONCLUSION}

The recent use of salivary biomarkers has changed the way fatigue has been measured. The development of the Fatigue Biomarker Index has brought stress and fatigue research to a new level with the ability to objectively quantify fatigue. The possibilities and potential applications for the FBI are endless. However, further testing and more conclusive studies still need to be conducted.

According to John Kalns, co-founder and vice president of Hyperion, Hyperion has been expanding rapidly in staff numbers and has received more than double its revenue of the previous year [22]. With more resources being channeled into this relatively untapped yet lucrative arena, rapid growth and further developments are to be expected.

Other than salivary biomarkers, there is the also possibility of looking into other non-invasive biomarkers of stress, such as sweat and heart rate variability. With all these poten- tial developments, it is likely that the near future will bring many advances in stress and fatigue research.

\section{CONFLICT OF INTEREST}

The authors confirm that this article content has no conflicts of interest.

\section{ACKNOWLEDGEMENTS}

None declared.

\section{REFERENCES}

[1] Michael DJ, Daugherty S, Santos A, Ruby BC, Kalns JE. Fatigue biomarker index: an objective salivary measure of fatigue level. Accid Anal Prev 2012; 45S: 68-73.

[2] Silverman MN, Heim CM, Nater UM, Marques AH, Sternberg EM. Neuroendocrine and immune contributors to fatigue. PM R 2010; 2(5): 338-46.

[3] Loh S, Lamond N, Dorrian J, Roach G, Dawson D. The validity of psychomotor vigilance tasks of less than 10-minute duration. Behav Res Methods 2004; 36(2): 339-46.

[4] Collado-Hidalgo A, Bower JE, Ganz PA, Cole SW, Irwin MR. Inflamamtory biomarkers for persistent fatigue in breast cancer survivors. Clin Cancer Res 2006 4; 12: 2759 - 66.

[5] Fletcher MA, Zeng XR, Maher K, et al. Biomarkers in chronic fatigue syndrome: evaluation of natural killer cell function and dipeptidyl peptidase IV/CD26. PLoS ONE 2010; 5(5): 1-7.

[6] Malamud D, Rodriguez-Chavez IR. Saliva as a diagnostic fluid. Dent Clin North Am 2011; 55(1): 159-78.

[7] Azman MY, Aziah D, Norhayati MN, Zahiruddin M. Stress and salivary biomarker among assistant medical officers in Ministry of Health (MOH) hospitals in Kelantan and Terengganu, Malaysia. Int J Collab Res Intern Med Public Health 2011; 3(8): 634-43.

[8] Kirschbaum C, Hellhammer DH. Salivary Cortisol. In: Fink G, Eds. Encyclopedia of Stress. San Diego: Academic Stress 2000; pp. 379-83.

[9] Salimetrics. Cortisol 2012 [cited 2012 May 12]; Available from: http: //www.salimetrics.com/my-spitresearch/analytes/cortisol.php.

[10] Hellhammer DH, Wüst S, Kudielka BM. Salivary cortisol as a biomarker in stress research. Psychoneuendocrinology 2009; 34: 163-71.

[11] Kudielka BM, Hellhammer DM, Wüst S. Why do we respond so differently? Reviewing determinants of human salivary cortisol responses to challenge Psychoneuroendocrinology 2009; 34: 2-18.

[12] Nater UM, Rohleder N. Salivary alpha-amylase as a non-invasive biomarker for the sympathetic nervous system: Current state of research. Psychoneuroendocrinology 2009; 34: 486-96.

[13] Rohleder N, Nater UM. Determinants of salivary $\alpha$-amylase in humans and methodological considerations. Psychoneuroendocrinology 2009; 34: 469-85.

[14] Bosch JA, Veerman ECI, de Geus EJ, Proctor GB. $\alpha$-Amylase as a reliable and convenient measure of sympathetic activity: don't start salivating just yet! . Psychoneuroendocrinology 2011; 36: 449-53.

[15] Pacqué P, Booth C, Dwyer D. Salivary immunoglobulin A (sIgA) as a biomarker of immune suppression following the combat fitness assessment. Research Report DSTO-RR-0236. Australia: Aeronautical and Maritime Research Laboratory 2002.

[16] Ruby BC, Gaskill SE, Heil DP, Sharkey BJ, Hansen K, Lankford DE. Changes in salivary IgA during arduous wildfire suppression relative to work shift length.Med Sci Sports Exerc 2002; 34: 195.

[17] MacLaughlin BW, Wang D, Noone AM, et al. Stress biomarkers in medical students participating in a mind body medicine skills program. Evid Based Complement Alternat Med 2011; 2011: 1-8.

[18] Biotechnology H. Biomarkers for fatigue. In: Biotechnology $\mathrm{H}$ editor. Texas: Hyperion Biotechnology. Available at: www.hyperio biotechnology.com/files/hyprion-fatigue-biomarkers.pdf

[19] Kalns JE, Michael DJ, inventor; Hyperion Biotechnology, assignee. Methods and compositions for biomarkers of fatigue, fitness and physical performance capacity. US Patent 20110077472 A1, 2011.

[20] Asprey D, Legge A. How to quantify fatigue like never before. USA The Bulletproof Executive 2011. 
[21] Olson EJ, Drage LA, Auger RR. Sleep deprivation, physician performance, and patient safety. Chest 2009; 136: 1389-96.
[22] Pack W. Your spit can tell you're tired. San Antonio: Express-News 2011.

Received: June 12, 2012

Revised: August 23, 2012

Accepted: August 28, 2012

(C) Yi and Moochhala; Licensee Bentham Open.

This is an open access article licensed under the terms of the Creative Commons Attribution Non-Commercial License (http://creativecommons.org/licenses/by-nc/3.0/) which permits unrestricted, non-commercial use, distribution and reproduction in any medium, provided the work is properly cited. 